\title{
Evolution of a Salmonella typhimurium infection in younger and older mice under caloric restriction
}

\author{
R. A. Jarillo-Luna ${ }^{1}$, G. Oliver-Aguillón ${ }^{1}$, V. Rivera-Aguilar ${ }^{2}$, R. Valdés-Ramos ${ }^{3}$, J. Pacheco-Yépez ${ }^{1}$, \\ E. Lara Padilla ${ }^{1}$ and R. Campos Rodríguez ${ }^{1}$ \\ ${ }^{1}$ Sección de Postgrado e Investigación, Escuela Superior de Medicina, Instituto Politécnico Nacional, México D.F., \\ ${ }^{2}$ Departamento de Microbiología, UBIPRO, FES-Iztacala, UNAM, Tlalnepantla, Estado de México and ${ }^{3}$ Center for \\ Research and Graduate Studies of Health Sciences, Faculty of Medicine, Universidad Autónoma Estado de México
}

The ageing of the world population has been one of the recent changes with the greatest impact on health issues. Evidence has been reported that caloric restriction $(\mathrm{CR})$ slows the deterioration of immune functions normally associated with ageing ${ }^{(1)}$. However, the evidence of this effect is still scarce, and contradictory reports exist, such as the recent publication asserting that CR actually diminishes intestinal IgA levels in young healthy mice ${ }^{(2,3)}$. The aim of the present study is to provide further evidence in this field by determining whether CR modifies the evolution of a Salmonella typhimuriuminfection in younger (6 months old) and older (12 months old) mice. Nine-week-old male Balb/c mice were obtained and fed a standard NHI-31 diet. Four groups were formed: two groups had daily ad libitum feeding until reaching (i) 6 months of age (AL6m) or (ii) 12 months of age (AL12m), and another two groups had alternate day ad libitum feeding until reaching (iii) 6 months of age $(\mathrm{CR} 6 \mathrm{~m})$ or (iv) 12 months of age (CR12m). All animals were infected with a sublethal dose of S. typhimurium. Two subgroups were formed within each group,one sacrificed at 7 days and the other at 14 days post infection. The bacterial content of feces was determined daily, while at 7 and 14 days post infection the total $\operatorname{sigA}(\mathrm{T}$-sIgA) and specific sIgA against S. typhimurium (S-sIgA) was quantified in intestinal fluid, and the colonization of Peyer's patches, spleen and liver was evaluated. Bacterial elimination in feces was greater at both 7 and 14 days in the older than younger mice with daily feeding, as well as in the older than younger mice with alternate day feeding (CR). Bacterial colonization of Peyer's patches and the liver was also greater in older than younger mice, but not in the spleen Fig 1. Although the basal T-sIgA was greater in the older than younger mice, at both 7 and 14 days post infection the older CR and AL animals had less T-sIgA than the respective younger ones. Likewise, at 7 and 14 days post infection the older CR and AL animals had less S-sIgA than the respective younger ones. Comparing the CR and AL groups, bacterial colonization of organs was greater in the CR6m than the AL6m group and less in the CR12m than the AL12m group. The T-sIgA was less in the CR6m than the AL6m group, and greater in the CR12m than the AL12m group. The S-sIgA was greater in the CR6m and CR12m groups than the corresponding AL groups, Fig 2. The results show that CR improves the immune response to $S$. typhimurium compared to AL feeding, but that $\mathrm{CR}$ does not prevent the deterioration of this immune response caused by ageing.

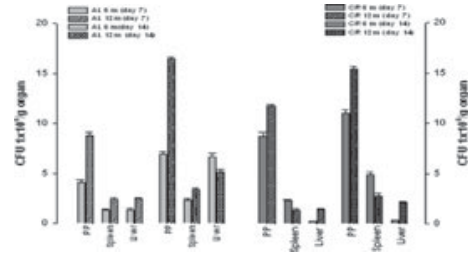

Figure 1. Bacterial colonization.

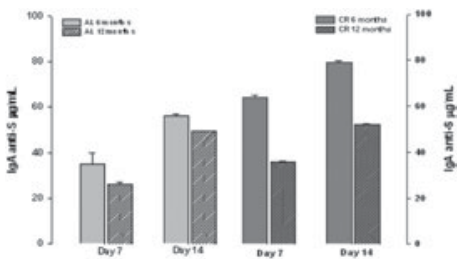

Figure 2. Levels IgA.

1. Massoro JE (2002) Elsevier Science B.V. Amsterdam. p. 73.

2. Lara-Padilla E, Campos-Rodriguez R et al. (2011) J Nutr Biochem 22 (6), 560-566.

3. Suárez-Souto MA, Lara-Padilla E et al. (2012) J Physiol Biochem 68 (2), 163-173. 